\title{
Dispersion-Enhanced Laser Gyroscope
}

\author{
David D. Smith ${ }^{* a, b}$, Hongrok Chang ${ }^{b}$, L. Arissian ${ }^{c}$, J. C. Diels ${ }^{c}$
}

${ }^{a}$ Spacecraft and Vehicle Systems Department, NASA Marshall Space Flight Center,

EV43, Huntsville, AL 35812

${ }^{b}$ Department of Physics, University of Alabama in Huntsville, 201B Optics Building, John Wright Drive, Huntsville, AL 35899

${ }^{\mathrm{c}}$ Department of Physics and Astronomy, University of New Mexico, 800 Yale Blvd., Albuquerque, NM 87131

(Dated: June 16, 2008)

We analyze the effect of a highly dispersive element placed inside a modulated optical cavity on the frequency and amplitude of the output modulation to determine the conditions for enhanced gyroscopic sensitivities. The element is treated as both a phase and amplitude filter, and the time-dependence of the cavity field is considered. Both atomic gases (two-level and multi-level) and optical resonators (single and coupled) are considered and compared as dispersive elements. We find that it is possible to simultaneously enhance the gyro scale factor sensitivity and suppress the dead band by using an element with anomalous dispersion that has greater loss at the carrier frequency than at the side-band frequencies, i.e., an element that simultaneously pushes and intensifies the perturbed cavity modes, e.g. a two-level absorber or an under-coupled 
optical resonator. The sensitivity enhancement is inversely proportional to the effective group index, becoming infinite at a group index of zero. However, the number of round trips required to reach a steady-state also becomes infinite when the group index is zero (or two). For even larger dispersions a steady-state cannot be achieved, and nonlinear dynamic effects such as bistability and periodic oscillations are predicted in the gyro response.

PACS Numbers: 42.50.Gy, 42.60.Da, 42.81.Pa

\section{INTRODUCTION}

The additional phase-shift acquired by an electromagnetic wave as a result of the motion of a medium, known as the Sagnac effect [1], can be measured directly by monitoring the amplitude of interferometer fringes, i.e., an interferometric optical gyroscope. Alternatively, the Sagnac phase-shift can be transformed into a frequency shift by using an optical cavity, i.e., a laser gyroscope (Fig. 1). The Doppler shift owing to the rotation of a ring laser, for example, is determined from the beat-frequency of two counter-propagating modes that are made to interfere at a location outside the cavity. In a laser gyroscope then, the rate of rotation is determined, not by direct measurement of the Sagnac phase-shift, but by measurement of the frequency shift associated with this phaseshift.

In recent years there has been interest in the development of highly dispersive materials whose resonant features can speed up, slow down, stop, store, or reverse the propagation of pulses of light [2-9] as a result of their substantially modified group velocities. The use of these modified group velocities for the enhancement of optical 
gyroscopes has been discussed by a variety of authors. [10-13] Leonhardt and Piwnitski have predicted an enhancement in the Fresnel drag coefficient for an interferometric gyro containing a slow-light medium. Shahriar et al. [14] point out that this enhancement applies only to relative rotation measurements, not to measurements of absolute rotation where the source and gyroscope are co-rotating and the Sagnac effect is independent of refractive index. As we have noted, the Sagnac effect is, however, often associated with a frequency shift, which in turn is highly sensitive to dispersion. Inside an optical cavity, the Sagnac phase shift accumulates each round trip and is transformed into a Doppler shift, and so it is possible to achieve a dispersion-related enhancement for the measurement of absolute motion in this case.

We are therefore obliged to consider the effect of such a highly dispersive element on the performance of a laser gyroscope in greater detail. This objective necessitates a timedependent analysis of the field inside the cavity because dispersion contributes a nonlinear feedback mechanism to the gyro response. Moreover, the absorptive part of the medium response cannot be neglected because it is related to the contrast of the beat-note with respect to backscattered frequencies which tend to reduce gyro sensitivities or even injection lock the modulation resulting in a dead band. In fact we will show that the absorptive part of the material response can be designed to mitigate such backscattering, suppressing the gyro dead band, while at the same time the dispersive part enhances the phase shift due to rotation. The rotation of the gyroscope is considered to be a strong modulation, and the theory of modulation spectroscopy is used to develop a timedependent analysis of the field in the cavity. Hence, we demonstrate that the dispersive element acts as an amplitude and phase nonlinear filter, shifting and demodulating the 
frequency components of the modulation, and we derive the conditions under which this modulation is enhanced. Our analysis agrees with previous mode pulling and intracavity spectroscopy experiments [15-20].

The geometry that we will consider is shown in Fig. 1, where a dispersive element, e.g., an atomic gas or optical micro-resonator, is incorporated inside the cavity of a ring laser gyroscope. All contributions to the dispersion within the cavity are lumped into a single "black box", that introduces a positive (or negative) feedback [21] in the form of an additional phase-shift $\Phi$, that is dependent on the original Sagnac phase-shift $\phi$, thereby increasing (or decreasing) the Doppler shift between the counter-propagating modes, and resulting in a modified beat-frequency.

\section{SAGNAC EFFECT AS A MODULATION}

Consider an arbitrary modulation of carrier frequency $\omega_{0}=c k_{0}$ and modulation frequency $\omega_{m}=c k_{m}$. Assume that any FM component of the signal is sufficiently weak that there are not more than two sidebands in the frequency spectrum, i.e., that the FM component is purely imaginary. The heterodyne beat-frequency and relative modulation amplitude of the interfering side-bands are known from the theory of modulation spectroscopy $[22,23]$ to be

$$
\omega_{b}(z, t) t=\omega_{m} t-\left[\frac{\varphi_{+}(z, t)-\varphi_{-}(z, t)}{2}\right],
$$

and 


$$
M(z, t)=\frac{\left[E_{+}(z, t)^{2}+E_{-}(z, t)^{2}\right]^{1 / 2}}{\sqrt{2} E_{0}(z, t)},
$$

respectively, where $\omega_{ \pm}=\omega_{0} \pm \omega_{m}=c k_{ \pm}$are the side-band frequencies, the quantities $E_{\nu}(z, t)$ and $\varphi_{\nu}(z, t)$ are slowly-varying amplitudes and phases, and frequency components are denoted by the subscript $\nu$, where $\nu=0,+$ or - . The modulation can be described by a parametric plot of the complex electric field amplitude as a function of time that traces out, at the frequency $\omega_{b}(z, t)$, an ellipse whose size is determined by the slowly-varying quantity $M(z, t)[23]$.

The relative modulation ellipse evolves with propagation through a dispersive medium. This evolution is already implicit in Eqs. (1) and (2), because the quantities $\omega_{b}(z, t)$ and $M(z, t)$ are slowly-varying in $z$ and $t$, but can be explicitly taken into account by the use of a complex transfer function $\tilde{\tau}(\omega)=\tau(\omega) \exp [i \Phi(\omega)]$, where $\Phi(\omega)$ is the effective phase shift, $\tau(\omega)$ is the transmittance, and capital Greek letters are used to distinguish effective quantities. Hence, the medium acts as both an amplitude and phase filter. The phase filter influences the beat-frequency, whereas the amplitude filter affects the relative modulation. For $p$ passes across the filter, the transfer function is

$$
\tilde{\tau}^{(p)}(\omega)=\left[\prod_{j=1}^{p} \tau^{(j)}(\omega)\right] \exp \left[i \sum_{j=1}^{p} \Phi^{(j)}(\omega)\right],
$$

where superscripts indicate the pass index. Equivalently, we make the substitutions $\varphi_{\nu}(z, t)=\varphi_{\nu}^{(0)}+\sum_{j=1}^{p} \Phi_{\nu}^{(j)}$ and $E_{\nu}(z, t)=E_{\nu}^{(0)} \prod_{j=1}^{p} \tau_{\nu}^{(j)}$, where $E_{\nu}^{(0)}$ and $\varphi_{\nu}^{(0)}$ are the initial amplitudes and phases, respectively, prior to interaction with the filter. Note that because 
the frequency components shift, their phase and amplitude change non-uniformly with each pass, until a steady-state is reached.

For a laser cavity containing a single dispersive element, $p=t / \tau_{c}$ is simply the number of round trips completed within time $t$. Applying Eq. (3) and differentiating Eq. (1) over $p_{0}$ round trips, we obtain the average effective beat-frequency and relative modulation amplitude

$$
\Omega_{b}^{(p)}=\omega_{m}-\frac{1}{2 \tau_{c} p_{0}} \sum_{j=p-p_{0}+1}^{p}\left[\Phi_{+}^{(j)}-\Phi_{-}^{(j)}\right],
$$

and

$$
M^{(p)}=\frac{1}{\sqrt{2}}\left[\left(\frac{E_{+}^{(0)}}{E_{0}^{(0)}} \prod_{j=1}^{p} \frac{\tau_{+}^{(j)}}{\tau_{0}^{(j)}}\right)^{2}+\left(\frac{E_{-}^{(0)}}{E_{0}^{(0)}} \prod_{j=1}^{p} \frac{\tau_{-}^{(j)}}{\tau_{0}^{(j)}}\right)^{2}\right]^{\frac{1}{2}},
$$

respectively, where $\tau_{c}$ is the cavity round trip time, and the $p=0$ term utilizes noneffective quantities, i.e., $\omega_{b}^{(0)}=\omega_{m}-\left(\varphi_{+}^{(0)}-\varphi_{-}^{(0)}\right) / 2 \tau_{c}$ is the beat-frequency prior to interacting with the dispersive element. The single-pass or "instantaneous" beatfrequency is obtained by setting $p_{0}=1$, i.e.,

$$
\Omega_{b}^{(p)}=\omega_{m}-\frac{\Phi_{+}^{(p)}-\Phi_{-}^{(p)}}{2 \tau_{c}}
$$

The actual beat frequency measurement averages over a number of round trips, $p_{0}$, determined by the detector bandwidth compared to the cavity free spectral range. The first term on the RHS of Eq. (4) is the Doppler shift due to the Sagnac effect itself, 
whereas the second term on the RHS of Eq. (4) and the products in Eq. (5) represent contributions from the dispersive medium. The dispersive contributions are determined by the effective phase difference and relative transmittance of the side-bands per round trip, respectively. Importantly, note that there is no influence of the dispersion on $\omega_{m}$, i.e., the Sagnac effect itself is intrinsically independent of refractive index. The dispersive contribution is strictly a consequence of the associated Doppler shift (it goes to zero when $\left.\Omega_{+}^{(j)}=\Omega_{-}^{(j)}\right)$.

As a consequence of the dispersion, these formulas are iterative: the effective phase shift and transmittance of any particular component are evaluated at the frequency of the previous pass, i.e., $\Phi^{(j)}=\Phi\left(\Omega^{(j-1)}\right)$ and $\tau^{(j)}=\tau\left(\Omega^{(j-1)}\right)$. They therefore depend on the history of beat-frequencies (back to when the side-bands first approach a material resonance). This is emphasized by the corresponding recursion relations

$$
\Omega_{b}^{(j)}\left(\omega_{m}^{(j)}, \Omega_{b}^{(j-1)}\right)=\omega_{m}^{(j)}+\Omega_{d}^{(j)}\left(\Omega_{b}^{(j-1)}\right)
$$

and

$$
M^{(j)}\left(\Omega_{b}^{(j-1)}, M^{(j-1)}\right)=\frac{\tau_{+}^{(j)}}{\tau_{0}^{(j)}} M^{(j-1)},
$$

where $\Omega_{d}^{(j)}\left(\Omega_{b}^{(j-1)}\right)=-\left[\Phi_{+}^{(j)}-\Phi_{-}^{(j)}\right] / 2 \tau_{c}=G^{(j)} \Omega_{b}^{(j-1)}$ and $\omega_{m}^{(j)}$ are again the dispersive and Sagnac contributions to the instantaneous beat-frequency, respectively, $G^{(j)}$ is the feedback gain [14], and we allow the Sagnac contribution to change on a time scale comparable to that of the dispersive propagation, i.e., $\omega_{m} \rightarrow \omega_{m}^{(j)}$, e.g., due to a changing rotation rate. Furthermore, in writing Eq. (8) we have assumed that the dispersive 
element is resonant with the unperturbed cavity such that $\tau_{+}^{(j)}=\tau_{-}^{(j)}$ and $E_{+}=E_{-}$. Note that the dispersion term $\Omega_{d}^{(j)}\left(\Omega_{b}^{(j-1)}\right)$ provides both the nonlinearity and feedback necessary for nonlinear dynamic behavior, such as bistability.

We model the counter-propagating modes of the rotating gyro by the two strong sidebands at $\Omega_{ \pm}$, such that $\Omega_{b}^{(p)}$ represents the laser gyro beat-frequency, measured at some location outside the cavity. Residual backscattering contributes a variety of central frequency components that fall between the side-bands. It is well-known that if the backscattering is sufficiently strong these central frequencies (which we model heuristically by the inclusion of a single weak carrier frequency) can injection lock the side bands resulting in a dead band in the gyro response (see Appendix A) [24]. The relative modulation $M^{(p)}$ indicates the strength of these central frequencies, and thus the size of the dead band. Therefore, to enhance the performance of a laser gyroscope, a dispersive element should be designed to increase both the beat-frequency (via the phase filter) and relative modulation (via the amplitude filter), i.e., it should increase both the rotation speed and size of the relative modulation ellipse, respectively.

\section{ENHANCEMENT OF GYRO SENSITIVITY}

It is clear from Eq. (7) that dispersion alters the gyro beat-frequency. Intuitively, this is not difficult to understand: In the absence of a dispersive medium, the red-shifted component of a rotating gyro accumulates more phase than the blue-shifted component, due its longer path length. But dispersion introduces an additional phase shift, whose size and sign is dependent on the original (Sagnac) phase-shift, and therefore can either enhance or compensate the measured beat-note, in a manner analogous to regenerative 
and degenerative feedback in electronic amplifiers [21]. When $\Phi_{+}^{(j)}<\Phi_{-}^{(j)}$, the Sagnac phase shift is augmented due to the positive feedback $\left(G^{(j)}>0\right)$, and the beat-frequency increases (the rotation of the modulation ellipse accelerates until it reaches some higher steady-state speed). Note that this condition requires the presence of anomalous dispersion, but the frequencies $\Omega_{ \pm}$may fall outside the region of anomalous dispersion. On the other hand, when $\Phi_{+}^{(j)}>\Phi_{-}^{(j)}$, the Sagnac phase shift is compensated by the negative feedback $\left(G^{(j)}<0\right)$ associated with the normal dispersion, thereby decreasing the beat-frequency.

An increase in the beat-frequency does not by itself, however, ensure an increase in gyro sensitivity. Rather, the gyro scale factor sensitivity is proportional to the derivative of Eq. (7),

$$
\frac{d \Omega_{b}^{(p)}}{d \omega_{m}^{(p)}}=1+S^{(p)} \frac{d \Omega_{b}^{(p-1)}}{d \omega_{m}^{(p)}}
$$

where

$$
S^{(p)} \equiv \frac{d \Omega_{d}^{(p)}}{d \Omega_{b}^{(p-1)}}=-\frac{1}{2 \tau_{c}} \frac{d\left(\Phi_{+}^{(p)}-\Phi_{-}^{(p)}\right)}{d \Omega_{b}^{(p-1)}}=-\frac{1}{2 \tau_{c}} \frac{d\left(\Phi_{+}^{(p)}+\Phi_{-}^{(p)}\right)}{d \omega}
$$

is the sensitivity gain. Now, let us define an effective group index of refraction ${ }^{g} N$ for the gyro cavity. First, we must define an effective propagation constant $K(\omega)=n_{c} \omega / c+\Phi(\omega) / L$, where $n_{c}$ is the index of refraction of the cavity medium, and $L$ is the size of the cavity, equivalent to the distance between dispersive elements. Then, 
ignoring all material dispersion except that of the element under consideration, the effective group index is

$$
{ }^{g} N(\omega)=c \frac{d K(\omega)}{d \omega}=n_{c}\left[1+\frac{1}{\tau_{c}} \frac{d \Phi(\omega)}{d \omega}\right]
$$

where $\tau_{c}=n_{c} L / c$, and $d \Phi / d \omega$ corresponds to the delay time of a long monochromatic pulse. Incorporating Eq. (11) into Eq. (10) yields

$$
S^{(p)}=1-\frac{{ }^{g} N_{a v g}^{(p)}}{n_{c}}=G^{(p)}+\Omega_{b}^{(p)} \frac{d G^{(p)}}{d \Omega_{b}^{(p-1)}},
$$

where ${ }^{g} N_{a v g}^{(p)}=\left({ }^{g} N_{+}^{(p)}+{ }^{g} N_{-}^{(p)}\right) / 2$ is the average group index of the side-bands. Note that in general the sensitivity and feedback gain are not equal, i.e., $S^{(p)}$ is always negative in regions of normal dispersion (because $d G^{(p)} / d \Omega_{b}^{(p-1)}$ is negative), but $G^{(p)}$ can be positive. When the dispersive element is resonant with the unperturbed cavity, the group index is symmetric about $\omega_{0}$, i.e., ${ }^{g} N_{+}^{(p)}={ }^{g} N_{-}^{(p)}$. In this case ${ }^{g} N_{a v g}^{(p)}={ }^{g} N_{+}^{(p)}={ }^{g} N_{-}^{(p)}$. Substitution of Eq. (12) into Eq. (9) yields,

$$
\frac{d \Omega_{b}^{(p-1)}}{d \omega_{m}^{(p)}}=\frac{1-d\left[\Omega_{b}^{(p)}-\Omega_{b}^{(p-1)}\right] / d \omega_{m}^{(p)}}{{ }^{g} N_{a v g}^{(p)} / n_{c}} \underset{\text { steadystate }}{\rightarrow} \frac{1}{{ }^{g} N_{a v g} / n_{c}} .
$$

Eq. (13) provides the evolution of the gyro scale factor with round trip as a function of the input modulation frequency $\omega_{m}^{(p)}$, given an element with group index ${ }^{g} N_{\text {avg }}^{(p)}$. In the steady-state, the beat-frequency and its sensitivity become independent of round trip, and $d \Omega_{b} / d \omega_{m}=n_{c} /{ }^{g} N_{a v g}$, in agreement with Shahriar et al. [14]. The same result is obtained even when the beat-frequency is changing, provided the group index remains 
constant, i.e., in linear dispersion spectral regions where $\Phi(\omega)$ varies approximately linearly with $\omega$, e.g., when the side-band frequencies never stray very far from resonance. In this near-resonance case, $S^{(p)} \approx G^{(p)}$, and $d \Omega_{b}^{(p)} / d \omega_{m}^{(p)} \approx \Omega_{b}^{(p)} / \omega_{m}^{(p)}$, i.e., an enhancement in beat-frequency corresponds to an enhancement in sensitivity. Note also that because the dispersion is typically largest on-resonance (an exception would be a Fano resonance), $d \Omega_{b}^{(p)} / d \omega_{m}^{(p)} \leq n_{c} /{ }^{g} N_{0}^{(p)}$ represents an upper bound on the gyro sensitivity.

Such a steady-state is only achieved for group indices within the range $0<{ }^{g} N_{\text {avg }}^{(p)} / n_{c}<2$, i.e., for $\left|S^{(p)}\right|>1$ nonlinear dynamic effects become evident. For ${ }^{g} N_{\text {avg }}^{(p)} / n_{c}<0$ bistability occurs due to strong positive feedback $\left(S^{(p)}>1\right)$, whereas for ${ }^{g} N_{\text {avg }}^{(p)} / n_{c} \geq 2$ continuous periodic oscillations arise in the beat-frequency as a result of strong negative feedback $\left(S^{(p)}<-1\right)$. Note that at ${ }^{g} N_{\text {avg }}^{(p)}=0$, Eq. (13) predicts a critical anomalous dispersion (CAD) where the gyro sensitivity becomes infinite. In practice, however, the gyro enhancement is limited by group-velocity dispersion [14], and because the number of round trips required to reach a steady-state increases with the dispersion, becoming infinite when ${ }^{g} N_{\text {avg }}^{(p)}=0$ (or ${ }^{g} N_{\text {avg }}^{(p)} / n_{c}=2$ ). Thus, even within the range $0<{ }^{g} N_{\text {avg }}^{(p)} / n_{c}<2$, a steady-state might not be attainable within a reasonable number of round trips, i.e., before a measurement is required. In this case, the sensitivity would be reduced slightly, by the factor $1-d\left(\Omega_{b}^{(p)}-\Omega_{b}^{(p-1)}\right) / d \omega_{m}^{(p)}$.

Generally, according to Eq. (9), an enhancement in sensitivity, $\left|d \Omega_{b}^{(p)} / d \omega_{b}^{(p)}\right|>1$, occurs when the sensitivity gain, scale-factor product satisfies either

$$
S^{(p)} \frac{d \Omega_{b}^{(p-1)}}{d \omega_{m}^{(p)}}>0
$$


or

$$
S^{(p)} \frac{d \Omega_{b}^{(p-1)}}{d \omega_{m}^{(p)}}<-2,
$$

i.e., for either normal or anomalous dispersion. However, when restricted to the steadystate, i.e., $0<{ }^{g} N_{a v g}^{(p)} / n_{c}<2$, Eq. (15) cannot be satisfied, and Eq. (14) is only satisfied when $0<{ }^{g} N_{a v g}^{(p)} / n_{c}<1$, i.e., for anomalous dispersion that is sufficiently weak. When the side-band frequencies $\Omega_{ \pm}^{(p)}$ do fall within a region of anomalous dispersion, satisfying Eq. (14), both the beat-frequency and its sensitivity are enhanced, but it is only when they are close to the resonance frequency of the dispersive element, $\omega_{r}$, i.e., in the central linear-dispersion region of the resonance, that the approximation $\Omega_{b}^{(p)} / \omega_{m}^{(p)} \approx d \Omega_{b}^{(p)} / d \omega_{m}^{(p)}$ is valid. In this region the enhancement in beat-frequency and its sensitivity are equal. But farther away from resonance, in regions of normal dispersion, the beat-note frequency is increased, but its sensitivity to rotation is reduced. Hence, it is possible to have enhanced beat frequencies as a result of positive feedback $\left(G^{(j)}>0\right)$, but reduced scale-factor sensitivies $\left(S^{(j)}<0\right)$ as a result of normal dispersion.

The change in beat-frequency may be understood simply as due to mode pulling or pushing by the dispersion. It is well known that the cold cavity modes of a laser are pulled towards the gain line (or pushed away from an absorbing line) by an amount that is proportional to their detuning (provided the detuning is small compared to the linewidth) and dependent on the strength of the dispersion [15-19]. In our case, the mode pulling effect is in addition to the frequency shift associated with the Sagnac effect, i.e., it 
modifies the perturbed cavity mode frequencies rather than the cold cavity modes. (In fact, the Doppler shift due to the Sagnac effect itself can also be thought of as due to mode pushing, where the phase-shift per round trip results from rotation rather than dispersion.) The phase-shift condition $\Omega_{ \pm, q} \tau_{c}+\varphi_{ \pm, q}+\Phi\left(\Omega_{ \pm, q}\right)=q 2 \pi$ yields the steadystate frequencies of the pulled modes,

$$
\Omega_{ \pm, q}=\omega_{ \pm, q}-\frac{\Phi\left(\Omega_{ \pm, q}\right)}{\tau_{c}} \approx \omega_{ \pm, q}\left[1 \mp\left(\frac{{ }^{g} N_{ \pm, q}}{n_{c}}-1\right)\right],
$$

where $q$ is an integer mode number, $\Phi\left(\Omega_{ \pm, q}\right)$ is the effective phase shift evaluated at the pulled frequencies, and $\omega_{ \pm, q}=\omega_{q}-\varphi_{ \pm, q} / \tau_{c}$ are the un-pulled mode frequencies of the perturbed cavity, Doppler-shifted from the cold cavity mode frequencies $\omega_{q}=q 2 \pi / \tau_{c}$ by the Sagnac phase-shift per round trip, $\varphi_{ \pm, q}$. For detunings small compared to the linewidth, the modes are pulled towards the resonance frequency $\omega_{r}$ for ${ }^{g} N_{ \pm, q}>1$ (e.g. an inverted two-level gain medium) and pushed away from $\omega_{r}$ for ${ }^{g} N_{ \pm, q}<1$ (e.g. a twolevel absorber). For larger detunings, the modes can be pushed away from the resonance even when ${ }^{g} N_{ \pm, q}>1$, though the sensitivity will not be enhanced.

For any one of the $q$ mode numbers, the pulled beat frequency, $\Omega_{b}=\left(\Omega_{+}-\Omega_{-}\right) / 2$, is then given by

$$
\Omega_{b}=\omega_{b}-\frac{\Delta \Phi\left(\Omega_{b}\right)}{2 \tau_{c}} \approx \omega_{b}\left[1-\left(\frac{{ }^{g} N_{a v g}}{n_{c}}-1\right) \frac{\Omega_{b}}{\omega_{b}}\right]
$$

where $\Delta \Phi\left(\Omega_{b}\right)=\Phi\left(\Omega_{+}\right)-\Phi\left(\Omega_{-}\right)$is the effective phase difference of the pulled modes, and $\omega_{b}=\left(\omega_{+}-\omega_{-}\right) / 2$ is the un-pulled beat-frequency. Taking the derivative of Eq. (17) 
yields $d \Omega_{b} / d \omega_{b}=1 /{ }^{g} N_{a v g} \approx \Omega_{b} / \omega_{b}$, in agreement with Eq. (13). The approximations shown are valid for mode frequencies within the central linear-dispersion region, i.e., close to the resonance frequency $\omega_{r}$. The derivation is a steady-state one, i.e., it does not keep track of the round trip $p$. But clearly the mode pulling or pushing is time-dependent. For larger dispersions, the steady-state analysis is no longer sufficient, and the nonlinear dynamics of the problem become evident. Notably, such nonlinear effects have been observed in high-gain lasers [18, 19].

\section{COMPARISON OF ATOMS AND RESONATORS}

These derivations are general and apply both to atomic media, and to optical resonators when the Sagnac effect in the resonator itself can be neglected, e.g., in the limit where the size of the resonator is zero, or the resonator is planar. We do not consider the Sagnac effect inside the micro-resonator itself, as this has been treated previously [11]. As we have seen, to properly understand the effect on the beat-note, such an element must be treated as both a phase and amplitude filter. The amplitude and filters can work in concert, or against one another. For non-inverted two-level atoms or under-coupled optical resonators, the absorption is higher on-resonance, which attenuates the carrier frequency more than the sidebands, increasing the relative modulation, while at the same time the anomalous dispersion increases the magnitude of the beat frequency. The opposite occurs if the two-level atom is inverted or the resonator is amplifying, i.e., the amplification is higher on-resonance, decreasing the relative modulation, and the dispersion is normal, which decreases the modulation frequency. Hence, in all of these cases the amplitude and phase filters work together. However, a particular distinction of 
optical resonators is that they can be non-amplifying and possess normal dispersion provided they are over-coupled (whereas two-level atoms must be inverted to possess normal dispersion on resonance), thereby increasing the relative modulation but decreasing the beat-frequency. In this case the amplitude and phase filters work against one another.

Similar considerations hold when the dispersion is modified via coherent coupling schemes, e.g. for multi-level atoms and coupled optical resonators. Consider three prototypical atomic coherence schemes: electromagnetically-induced absorption (EIA) [25], electromagnetically-induced transparency (EIT) [26], and gain-assisted superluminality (GAS) [27, 28] from a double-gain line. The cases of EIA and GAS are similar to an absorbing two-level atom, i.e., greater carrier frequency absorption increases the relative modulation, while the associated anomalous dispersion increases the magnitude of the beat frequency. For the case of EIT, on the other hand, the transparency and normal dispersion on-resonance result in both decreased relative modulation and beat frequency, similar to an inverted two-level atom. In all these atomic coherence schemes the amplitude and phase filters work together. Again, to find cases where the amplitude and phase filters oppose one another, one must turn to the analogous coherence effects in coupled resonators [7-9].

\section{RESULTS}

For the dispersive element, we utilize optical resonators, because both anomalous and normal dispersion can be readily examined without the need for gain, simply by changing from under-coupled (anomalous dispersion) to over-coupled (normal dispersion), and 
because resonators offer all of the coherence effects available in atomic media, but in a more robust all-solid-state configuration. We assume a carrier frequency of $\omega_{0} / 2 \pi=10^{14} \mathrm{~Hz}$, a cavity with a free spectral range of $1 / \tau_{c}=100 \mathrm{MHz}$, and that the resonator is resonant with the unperturbed cavity, i.e., $\omega_{r}=\omega_{0}$.

We investigate four regimes of group indices: a) weak anomalous dispersion, $0<{ }^{g} N_{a v g}^{(p)} / n_{c}<1$, b) weak normal dispersion, $1<{ }^{g} N_{a v g}^{(p)} / n_{c}<2$, c) strong anomalous dispersion ${ }^{g} N_{a v g}^{(p)} / n_{c}<0$, and d) strong normal dispersion ${ }^{g} N_{a v g}^{(p)} / n_{c}>2$. The free spectral range of the resonator is held fixed at $1 / \tau_{r}=100 \mathrm{GHz}$, and the group index is varied by changing the coupling and loss of the resonator, while leaving the cavity parameters fixed. In Fig. 2 and 3 the resonator quality factor is (a) $Q=4 \times 10^{6}$ and (b) $Q=10^{6}$, respectively, whereas in Figs. 4 and 5, the quality factor is $Q=10^{6}$ for each of the four cases, corresponding to a $100 \mathrm{MHz}$ FWHM resonance linewidth. The maximum group indices are a) ${ }^{g} N_{0}^{(p)} / n_{c}=0.2$, b) ${ }^{g} N_{0}^{(p)} / n_{c}=1.8$, c) ${ }^{g} N_{0}^{(p)} / n_{c}=-1.0$, and d) ${ }^{g} N_{0}^{(p)} / n_{c}=3$, respectively, such that the magnitude of the sensitivity gain is $\left|S^{(p)}\right|=0.8$ for cases (a) and (b) and $\left|S^{(p)}\right|=2$ for cases (c) and (d). In Figs. 2 and 3 we plot only the cases of weak dispersion, i.e., we only consider dispersions that result in a steady-state in the beat-frequency, whereas in Figs. 4 and 5 all four cases are plotted.

In Figs. 2 and 3, the initial modulation frequency is set to $\omega_{m}^{(0)} / 2 \pi=100 \mathrm{KHz}$ and the beat-frequency is allowed to evolve over 50,000 round trips to a steady-state, whereas in Figs. 4 and 5, the modulation frequency is changed in increments of $\Delta \omega_{m} / 2 \pi=100 \mathrm{KHz}$ every $\Delta p=50$ round trips, beginning at a frequency far from resonance, $\omega_{m}^{(0)} / 2 \pi=-100 \mathrm{MHz}$, increasing to $\omega_{m}^{(100000)} / 2 \pi=100 \mathrm{MHz}$, and then decreasing again back to the initial frequency, $\omega_{m}^{(200000)} / 2 \pi=-100 \mathrm{MHz}$ to form a complete loop after a 
total of 200,000 round trips. So, over the course of this loop, there is always more than one effective phase-shift per modulation cycle.

In Fig. 2, the counter-propagating modes are plotted in the frequency domain, after they have reached the steady-state, with and without the resonators present in the cavity. A weak carrier frequency is included as a heuristic, to account for backscattering frequencies. A time-domain graph of the beat-frequency and relative modulation is presented in Fig. 3. These figures show how the modulation side-bands are pushed (pulled) by the anomalous (normal) dispersion, eventually reaching their steady-state values of $\Omega_{ \pm}^{(\infty)} / \omega_{m}^{(0)} \approx n_{c} /{ }^{g} N_{0}^{(p)}$, after no more than a few 10's of round trips. In Fig. 3b, oscillations occur as a result of some over-pulling, prior to reaching the steady-state. The number and amplitude of these oscillations increase with the group index. Unlike the beat-frequency, the relative modulation does not reach a steady-state, but continues increasing to infinity. This suppression of the central frequencies in comparison to the side-band frequencies, strengthens the beat-note contrast and suppresses the gyro dead band.

In Fig. 4, the effective beat frequency is plotted every 50 round trips (which we know from Fig. 3 is a sufficient number of round trips to reach a steady-state, if one can be achieved), as the initial modulation frequency is varied for the four regimes of dispersion. The slope of these curves at any point is determined by Eq. (9). In (a) and (b) the gyro scale factor is modified by the factor $n_{c} /{ }^{g} N_{0}^{(p)}$ at resonance. In (c) and (d) the strong dispersion leads to bistability and continuous periodic oscillations, respectively.

Fig. 5 is simply a zoomed-in version of Fig. 4 near zero beat-frequency, i.e., for sideband frequencies close to the resonance frequency $\omega_{r}$. The plot resolution is 1 round trip 
rather than 50, so that time-dependent effects can be more readily resolved. Note that the dispersion causes deviations from perfectly sharp steps of $\left(\Omega_{b}^{(\Delta p+p)}-\Omega_{b}^{(p)}\right)=\Delta \omega_{m}$. Closer to resonance, these deviations becomes more pronounced, and the beat-frequency takes more round trips to reach a steady-state (the relative modulation also takes more round trips to increase). In (a) and (b) the dispersion is still sufficiently weak that the beat-frequency reaches steady-state values given by $\left(\Omega_{b}^{(\Delta p+p)}-\Omega_{b}^{(p)}\right) / \Delta \omega_{m} \approx n_{c} /{ }^{g} N_{0}^{(p)}$. In (c) and (d), on the other hand, the nonlinear effects associated with the dispersion are so large that a steady-state cannot be reached. In Fig 5c, the zero in beat-frequency is pushed to a larger value of $p$ as a result of hysteresis, where a jump is observed, which results in bistability. In Fig. 5d, the dispersion is strong enough to drive continuous oscillations, whose amplitude is given by $\left(\Omega_{b}^{(\Delta p+p)}-\Omega_{b}^{(p)}\right)^{g} N_{a v g}^{(p)} / 2 n_{c}$.

\section{SUMMARY AND CONCLUSION}

In summary, we find that for two-level atoms or under-coupled optical resonators incorporated into a laser gyro cavity, the amplitude and phase filters work together to enhance the modulation, simultaneously enhancing the gyro scale factor via the anomalous dispersion and suppressing the dead band via the absorption. In fact, we could have used a single complex phase shift $\tilde{\Phi}(\omega)$ to describe the filter, that when incorporated into the cavity gives rise to a complex effective group index ${ }^{g} \tilde{N}(\omega)$ and feedback $\tilde{G}(\omega)$, which in turn modify the complex beat-frequency $\tilde{\Omega}_{b}$, which consists of the real-valued beat frequency and its contrast. Anomalous (normal) dispersion and absorption (gain) provides a positive (negative) complex feedback. The scale-factor sensitivity is inversely proportional to the effective group index, becoming infinite at a 
group index of zero, yet requiring an infinite number of round trips to reach steady-state at this point. In fact, the time-dependency is more likely to limit the achievable performance of a dispersion-enhanced gyro than higher-order dispersion. Steady-state results are only obtained for $0<{ }^{g} N_{\text {avg }}^{(p)} / n_{c}<2$. For ${ }^{g} N_{\text {avg }}^{(p)}<0$ and ${ }^{g} N_{\text {avg }}^{(p)}>2$ bistability and continuous oscillations are predicted, respectively. The enhancement of gyro sensitivity should be easier to observe for Doppler-broadened atomic gases than for micro-resonators, owing to the velocity distribution.

Furthermore, our analysis shows that not only can a dispersive element enhance desirable phase shifts (e.g. due to rotation), but it can also compensate deleterious phase shifts (e.g., due to a cavity instability), as shown in Fig. 4b. The resonances of inverted two-level atoms or amplifying micro-resonators should, therefore, be useful for laser selfstabilization, simultaneously reducing frequency and amplitude fluctuations, without the need for extra-cavity feedback. In fact, mode-pulling has long been demonstrated as a means for laser self-stabilization [29], but the development of highly dispersive media, employed separate from the gain medium, make these ideas more promising. Indeed, efficient noise or dead band suppression depends upon materials with stable high- $Q$ resonances.

The introduction of such a dispersive element into the cavity will increase spontaneous emission noise. To minimize the increase in pumping and avoid group indices where nonlinear dynamics occur, narrow yet shallow resonances (away from critical coupling) should be employed. Note that Eq. (7) can readily be separated into signal and noise contributions. We have examined the effect of dispersion on the bandwidth of a white noise input and have found that the noise bandwidth increases at a 
rate considerably slower than the inverse of the group index. The reason for this is that for a noisy input that changes each round trip, a steady-state is never reached. Away from the steady-state, the beat-frequency depends on the history of beat-frequencies, i.e., is path dependent. Therefore, the enhancement of a white noise input is different (in fact, substantially lower) than that of a monotonic signal. Hence, the dispersive medium filters out high frequency noise in comparison to the signal. Another way to look at this is that the noise adds a finite bandwidth to the beat-note, and this bandwidth is increased by the dispersion, but slower than the inverse of the group index. A similar result is found in the calculations of Appendix A, where it is shown that the regenerative gain linewidth remains finite even for a group index of zero.

We note, finally, the analogy between dispersive and nonlinear optical elements: Just as the saturation of a laser gain medium compensates intensity variations, a dispersive element can compensate phase variations. Fast-light media increase phase variations and are therefore analogous to saturable absorbers, which increase the amplitude noise of a laser. Slow light media, on the other hand, decrease phase noise, and are analogous to two-photon absorbers, which decrease amplitude noise. For both nonlinear optical and dispersive elements, when the system is driven beyond its ability to assume a steadystate, nonlinear dynamic effects such as bistability can occur.

\section{ACKNOWLEDGEMENTS}

The authors acknowledge support from NASA Marshall Space Flight Center Institutional Research and Development Grants CDDF03-17 and CDDF04-08, and the 
United Negro College Fund Office of Special Programs. This work was also supported by NSF ECS 0601612.

\section{APPENDIX A: GYRO LOCK-IN CONDITIONS}

Let us treat the laser cavity as a regenerative amplifier below threshold, having only one port for input and output. The complex transfer function for the electric-field is

$$
\tilde{\mathcal{G}}(\omega)=\frac{\tilde{E}_{\text {out }}}{\tilde{E}_{\text {in }}}=\frac{\tilde{R}-g \tilde{\tau}(\omega) \exp (i \phi)}{1-\tilde{R} g \tilde{\tau}(\omega) \exp (i \phi)} \approx \frac{R-g \tau(\omega)}{1-\operatorname{Rg} \tau(\omega)(1+i[\phi+\Phi(\omega)])},
$$

where $\phi=\left(\omega-\omega_{0}\right) \tau_{c}$ is the single-pass phase-shift, $g$ is the round trip gain, $\tilde{R}=R \exp \left(i \varphi_{R}\right)$ is the complex port reflectivity, and $\operatorname{Rg} \tau(\omega)$ is the net gain per round trip. We have assumed the port reflectivity is real $\left(\varphi_{R}=0\right)$, small detunings $(\phi+\Phi(\omega) \approx 0)$ from resonance, and that there is some strong frequency-dependence near the resonance frequency $\omega_{0}$, e.g., due to some high- $Q$ filter or hole burning in the gain line, such that we must include the complex filter function $\tilde{\tau}(\omega)=\tau(\omega) \exp [i \Phi(\omega)]$

Now, the net gain at the free-running oscillation frequency $\omega_{0}$ is clamped at the threshold value $\operatorname{Rg} \tau\left(\omega_{0}\right)=1$. Hence, the gain at some other, injected, frequency is $\operatorname{Rg} \tau(\omega)=\operatorname{Rg} \tau\left(\omega_{0}\right) x(\omega)=x(\omega)$, where $x(\omega) \equiv \tau(\omega) / \tau\left(\omega_{0}\right)=\exp \left[-\alpha_{d}(\omega) \tau_{c} / 2\right]$ is the transmittance ratio, and $\alpha_{d}(\omega) \equiv \alpha(\omega)-\alpha\left(\omega_{0}\right)$ is the loss coefficient difference at the two frequencies. For small detunings the spectrum is approximately linear, so that the feedback and sensitivity gains are equal, i.e., $\Phi(\omega)=-G(\omega) \phi \approx[d \Phi(\omega) / d \phi] \phi=-S(\omega) \phi$. Hence, $\phi+\Phi(\omega) \approx{ }^{g} N \phi$, where the 
group index ${ }^{g} N$ is approximately independent of frequency. Within this constant groupindex approximation, the intensity amplification is then

$$
|\tilde{\mathcal{G}}(\omega)|^{2} \approx \frac{\left[x(\omega)-R^{2}\right]^{2}}{[1-x(\omega)]^{2}+\left[x(\omega)^{g} N \phi\right]^{2}} .
$$

The condition for lock-in of the free-running oscillation at frequency $\omega_{0}$ and intensity $I_{0}$, by an external injected signal at frequency $\omega_{1}$ and intensity $I_{1}$, is that the output intensity $\left|\tilde{G}\left(\omega_{1}\right)\right|^{2} I_{1}$ exceeds $I_{0}$, i.e.,

$$
\left[\phi_{1}\right]^{2} \leq\left[\frac{x\left(\omega_{1}\right)-R^{2}}{{ }^{g} N x\left(\omega_{1}\right) R}\right]^{2} \frac{I_{1}}{I_{0}}-\left[\frac{x\left(\omega_{1}\right)-1}{{ }^{g} N x\left(\omega_{1}\right)}\right]^{2} .
$$

Without the dispersive filter, i.e., $\tilde{\tau}\left(\omega_{1}\right)=x\left(\omega_{1}\right)={ }^{g} N=1$, we obtain the well-known formula for the injection lock-in frequency detuning,

$$
\left|\omega_{1}-\omega_{0}\right| \leq \frac{\left(1-R^{2}\right) / R}{\tau_{c}} \sqrt{\frac{I_{1}}{I_{0}}} \approx \frac{1}{\tau_{d}} \sqrt{\frac{I_{1}}{I_{0}}}
$$

where $\tau_{d}$ is the cavity lifetime. The lock-in frequency range is then twice this detuning, i.e., $\Delta \omega=2\left|\omega_{1}-\omega_{0}\right|$. The dispersive filter, however, alters the injection lock-in frequency, as a result of modification of the regenerative gain line. A plot of Eq. (19) is shown in Fig. 6, for various values of ${ }^{g} N$. For anomalous dispersions such that $\left|{ }^{g} N\right|<1$, the phase filter broadens the regenerative gain line, thereby increasing the lock-in condition. The maximum linewidth and lock-in range occur for ${ }^{g} N=0$. On the other 
hand, for stronger anomalous dispersions such that ${ }^{g} N<-1$, or for normal dispersions, i.e., ${ }^{g} N>1$, the phase filter narrows the gain line, decreasing the lock-in condition.

Now, in a laser gyro a small portion of the internal intensity, $\rho I_{0} /(1-R)$, is scattered into the counter-propagating beam, where $\rho$ is the scattering coefficient per round trip. This internal injection corresponds to an external injection of $\rho I_{0} /(1-R)^{2}=I_{1}$. Substituting into Eq. (20), we obtain the transcendental equation for the gyro lock-in range, i.e., the dead band,

$$
\left[\phi_{1}\right]^{2} \leq \frac{1}{{ }^{g} N^{2}}\left[\left(\frac{1-R^{2} / x\left(\omega_{1}\right)}{1-R}\right)^{2} \frac{\rho}{R}-\left(1-\frac{1}{x\left(\omega_{1}\right)}\right)^{2}\right] \approx\left(\frac{\gamma\left(\omega_{1}\right)}{\gamma_{d}} \frac{\sqrt{\rho}}{{ }^{g} N}\right)^{2}-\left(\frac{\alpha_{d}\left(\omega_{1}\right)}{2} \frac{\tau_{c}}{{ }^{g} N}\right)^{2},
$$

where $\gamma\left(\omega_{1}\right)=\gamma_{d}-\alpha_{d}\left(\omega_{1}\right) / 2=-\left(1 / \tau_{c}\right) \ln \left[R^{2} / x\left(\omega_{1}\right)\right]$ and $\gamma_{d}=1 / \tau_{d}=-\left(1 / \tau_{c}\right) \ln R^{2}$ are frequency dependent and independent cavity loss rates, i.e., with and without the dispersive filter, respectively, and the approximation holds in the limit of small cavity couplings $\left(R \approx 1, \gamma_{0} \approx 0\right)$, and differential losses $\left(x \approx 1, \alpha_{d} \approx 0\right)$. Again, in the absence of the dispersive filter, we recover the well-known formula for the gyro dead band,

$$
\Delta \omega=\frac{\sqrt{\rho}}{\tau_{c}}
$$

To determine how the dead band is altered by the amplitude and phase response of the dispersive medium, i.e., by $x$ and ${ }^{g} N$, respectively, we must solve Eq. (22), which is transcendental. In Fig. 7, the intersections of the right and left hand sides of Eq. (22) are plotted versus the phase detuning $\phi$ in dispersion-free dead band units, $\sqrt{\rho}$. Again, in comparison with the dispersion-free case, the dead band is decreased for $\left|{ }^{g} N\right|>1$, 
corresponding to narrowing of the regenerative gain linewidth, and increased for $\left|{ }^{g} N\right|<1$. Now, provided the dead band detuning is smaller than a critical detuning, found from the solution of the transcendental equation,

$$
\tau_{c}\left[\frac{\gamma_{d} \alpha_{d}\left(\omega_{1}\right)}{2 \gamma\left(\omega_{1}\right)}\right]<\sqrt{\rho},
$$

then differential absorption can be neglected, and the dead band can be approximated by

$$
\Delta \omega \approx \frac{\sqrt{\rho} / \tau_{c}}{\left|{ }^{g} N\right|} .
$$

Within this approximation of constant loss, any enhancement in beat-frequency is countered by a concomitant increase in the dead band, because they both scale as $1 /{ }^{g} N$. This is certainly the case for the lower curves of Fig. 7. Here, the dead band is considerably smaller than the critical detuning (which marks the strong departure of the RHS of Eq. (22) from the straight line), so that we can neglect the effect of differential absorption. Hence, in this case, the dead band is well approximated by Eq. (25). In contrast, for the upper two curves of Fig. 7, the dead band is large enough that the approximation fails, and differential absorption results in considerable suppression of the dead band. The suppression of the dead band near ${ }^{g} N=0$ by differential absorption is emphasized in Fig. 8, where solutions of Eq. (22) are directly compared with the approximations of Eqs. (24) and (25), and is clearly related to the fact that the line-width of the regenerative gain increases (due to the phase response) but does not become infinite at ${ }^{g} N=0$. 
We have assumed in this discussion that the dead band is much smaller than the resonator spectrum such that the approximation of constant group index holds. For dead bands comparable to the resonance linewidth, the reduction in dispersion in the wings of the resonance will tend to widen the dead band for normal dispersion and reduce it for anomalous dispersion.

\section{REFERENCES}

1. M. Sagnac, C. R. Acad. Sci. 157, 707 (1913).

2. K. J. Boller, A. Imamoglu, and S. E. Harris, Phys. Rev. Lett. 66, 2593 (1991).

3. M. D. Lukin, S. F. Yelin, and M. Fleischhauer, Phys. Rev. Lett. 84, 4232 (2000).

4. D. F. Phillips, A. Fleischhauer, A. Mair, R. L. Walsworth and M. D. Lukin, Phys. Rev. Lett. 86, 783 (2001).

5. M. F. Yanik and S. Fan, Phys. Rev. Lett. 92, 083901 (2004).

6. G. M. Gehring, A. Schweinsberg, C. Barsi, N. Kostinski and R. W. Boyd, Science 312, 895 (2006).

7. D. D. Smith, H. Chang, K. A. Fuller, and R. W. Boyd, Phys. Rev. A 69, 63804 (2004).

8. D. D. Smith and H. Chang, J. Mod. Opt. 51, 2503 (2005).

9. H. Chang and D. D. Smith, J. Opt. Soc. Am. B 22, 2237 (2005).

10. U. Leonhardt and P. Piwnitski, Phys. Rev. A 62, 055801 (2000).

11. A. B. Matsko, A. A. Savchenkov, V. S. Ilchenko and L. Maleki, Opt. Commun. 233, 107 (2004). 
12. B. Z. Steinberg, Phys. Rev. E 71, 056621 (2005).

13. J. Scheuer and A. Yariv, Phys. Rev. Lett. 96, 053901, (2006).

14. M. S. Shahriar, G.S. Pati, R. Tripathi, V. Gopal, M. Messall, and K. Salit, Phys. Rev. A 75, 053807 (2007).

15. W. R. Bennett, Phys. Rev. 126, 580 (1962).

16. L. Casperson and A. Yariv, Appl. Phys. Lett. 17, 259 (1970).

17. R. S. Gioggia, N. B. Abraham, Phys. Rev. A 29, 1304 (1984).

18. R. A. McFarlane, Phys. Rev. 135, A543 (1964).

19. B. K. Garside, IEEE J. Quant. Elect. QE-5, 97 (1969).

20. S. A. Diddams, J.C. Diels, and B. Atherton, Phys. Rev. A 58, 2252 (1998).

21. P. Horowitz and W. Hill, The Art of Electronics, (Cambridge Univ. Press, 1989), p. 175-306; H. V. Malmstadt, C. G. Enke, and E. C. Toren Jr., Electronics for Scientists, (W. A. Benjamin, Inc., New York, 1963).

22. G. C. Bjorklund, Opt. Lett. 5, 15 (1980).

23. M. A. Kramer, R. W. Boyd, L. W. Hillman, and C. R. Stroud Jr., J. Opt. Soc. Am. B 2, 1444 (1985).

24. F. Aronowitz, in Laser Applications, edited by M. Ross (Academic, New York, 1971), p. 113-200.

25. A. M. Akulshin, S. Barreiro, and A. Lezama, Phys. Rev. A 57, 2996 (1998).

26. S. E. Harris, J. E. Field, and A. Imamoglu, Phys. Rev. Lett. 64, 1107 (1990).

27. L. J. Wang, A. Kuzmich, and A. Dogariu, Nature 406, 277 (2000).

28. A. Dogariu, A. Kuzmich, and L. J. Wang, Phys. Rev. A 63, 53806 (2001). 
29. P. W. Smith and P. J. Maloney, Appl. Phys. Lett. 22, 667 (1973). 


\section{FIGURES}

Figure 1. (Color online) A dispersive element (represented by the box) inside a ring laser gyroscope introduces a feedback in the form of an additional phaseshift $\Phi$, that is dependent on the Sagnac phase-shift $\phi$, thereby modifying the Doppler shift between the counter-propagating modes, and resulting in a modified beat-frequency $\left(\Omega_{+}-\Omega_{-}\right) / 2$. In the absence of dispersion the beat frequency is simply $\left(\omega_{+}-\omega_{-}\right) / 2$.

Figure 2. Mode pushing (a) and mode pulling (b) of the counter-propagating laser gyro frequencies by weak (a) anomalous and (b) normal dispersion. The modes are shown $(i)$ without and $(i i)$ with the resonator placed inside the cavity, after reaching their steady-state frequencies. The effective phase shift and transmittance are also plotted for comparison. In (a), the carrier frequency is significantly attenuated, indicating suppression of backscattering frequencies and the gyro dead band.

Figure 3. Evolution of the beat frequency and relative modulation with cavity round trip for a cavity containing weak (a) anomalous and (b) normal dispersion. In (a) the beat-frequency increases due to mode pushing, whereas in (b) it undergoes oscillations and decreases as a result of mode pulling. In both cases the relative modulation increases due to greater carrier frequency 
absorption, but it increases sooner in (a) because the sidebands are pushed away from the carrier and because the $Q$ is larger.

Figure 4. Four regimes of gyroscopic response for a laser gyro containing a dispersive element: (a) increased scale factor sensitivity due to weak anomalous dispersion, (b) decreased scale factor sensitivity due to weak normal dispersion (c) bistability due to strong anomalous dispersion, and (d) bifurcation to periodic oscillations due to strong normal dispersion. The dotted line is the gyro response in the absence of the dispersive element (and in the absence of lock-in). The dashed line represents the upper limit on the sensitivity enhancement $1 /{ }^{g} N_{0}^{(p)}$.

Figure 5. Evolution of the beat frequency with cavity round trip for (a) weak anomalous dispersion, (b) weak normal dispersion, (c) strong anomalous dispersion, and (d) strong normal dispersion. The modulation frequency is changed in increments of $\Delta \omega_{m}=100 \mathrm{KHz}$ every $\Delta p=50$ round trips. But the dispersion causes deviation from a perfectly sharp step of $\left(\Omega_{b}^{(\Delta p+p)}-\Omega_{b}^{(p)}\right)=\Delta \omega_{m} . \quad$ In $\quad$ (a) $\quad\left(\Omega_{b}^{(\Delta p+p)}-\Omega_{b}^{(p)}\right)>\Delta \omega_{m}$ due to mode pushing, and in (b) $\left(\Omega_{b}^{(\Delta p+p)}-\Omega_{b}^{(p)}\right)<\Delta \omega_{m}$ due to mode pulling. The nonlinear effects are so large in (c) and (d), that a steady-state cannot be reached, resulting in bistability and continuous oscillations, respectively. 
Figure 6. (Color online) Regenerative gain line for $R=0.95$, and no dispersion (solid curve), i.e., ${ }^{g} N=1$, normal dispersion (dashed curve), i.e., ${ }^{g} N=2$, and anomalous dispersion (dashed curves), i.e., (a) ${ }^{g} N=0$, (b) ${ }^{g} N=0.5$, and (c) ${ }^{g} N=-2$. At ${ }^{g} N=0$ the line is broad (but not infinite), and narrows as ${ }^{g} N$ increases or decreases. Note that the widths for ${ }^{g} N=-2$ and ${ }^{g} N=2$ are equal. The gain deviates from unity for large detunings because of the small angle approximation.

Figure 7. (Color online) The dead band of a laser gryo with $R=0.95$ and $\rho=0.001$, shown as the intersections of the LHS. (two solid vertical curves) and RHS (solid curves) of Eq. (22). (a) ${ }^{g} N= \pm 0.02$, (b) ${ }^{g} N= \pm 0.1$, (c) ${ }^{g} N= \pm 0.5$, and $(d){ }^{g} N= \pm 2$. The positive group index is the wider of the two curves in each case. The dashed horizontal lines represent the constant-loss approximation of Eq. (25). The dead band is well approximated in (c) and (d), but in (a) and (b) the dead band is smaller than the approximation as a result of differential absorption. Increasing the scattering coefficient pushes the solid curves upward, thereby increasing the dead band.

Figure 8. Suppression of the dead band of a laser gyro with $R=0.95$ and $\rho=0.001$ by differential absorption. The open symbols are solutions of Eq. (22), and the crosses are solutions of Eq. (24). For large group indices, the dead band is well approximated by the constant-loss approximation, indicated by the solid curve, but for smaller group indices near ${ }^{g} N=0$, the dead band is 
suppressed by differential absorption, and is better approximated by Eq. (24) 


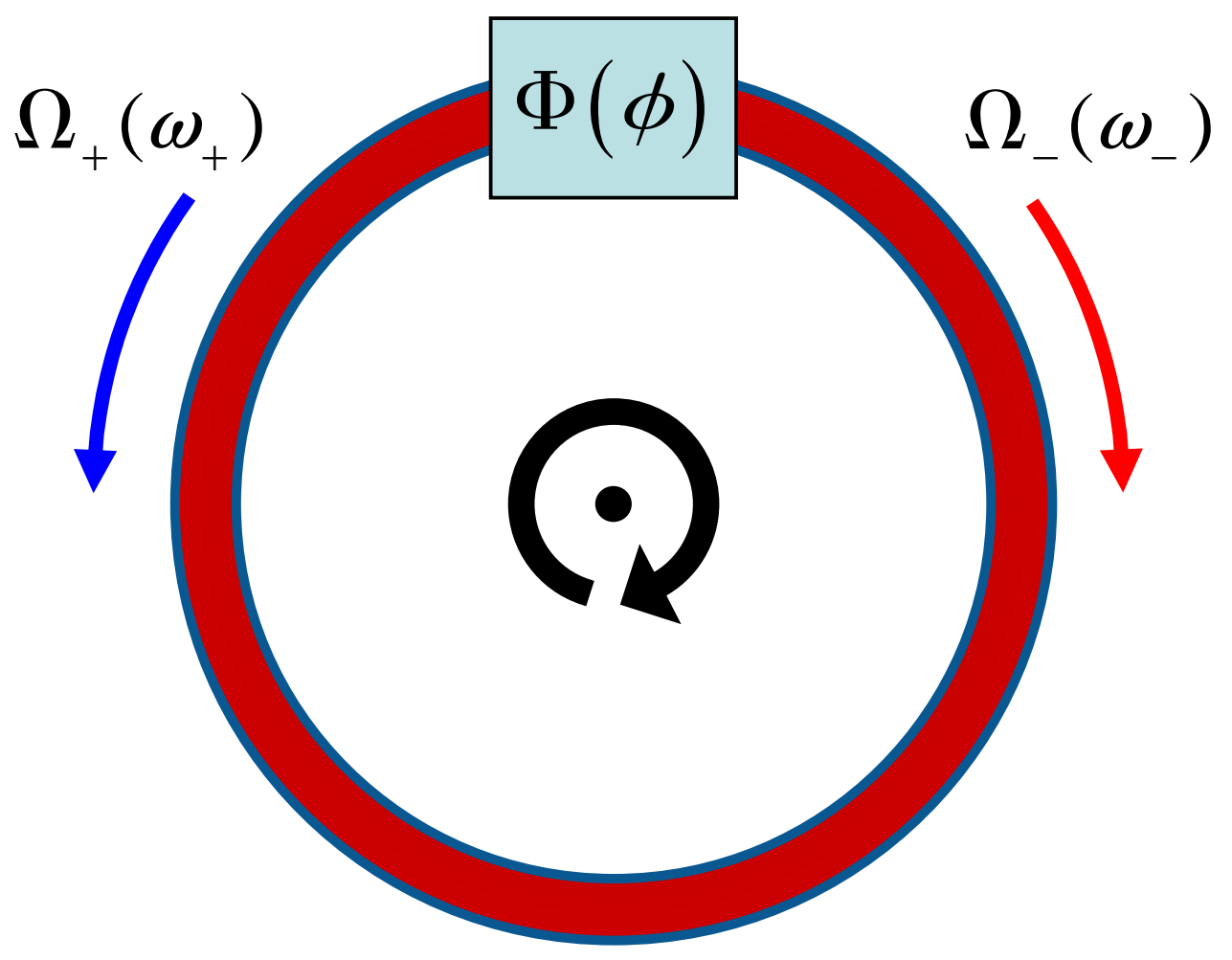

Figure 1 (color online) 
(a)

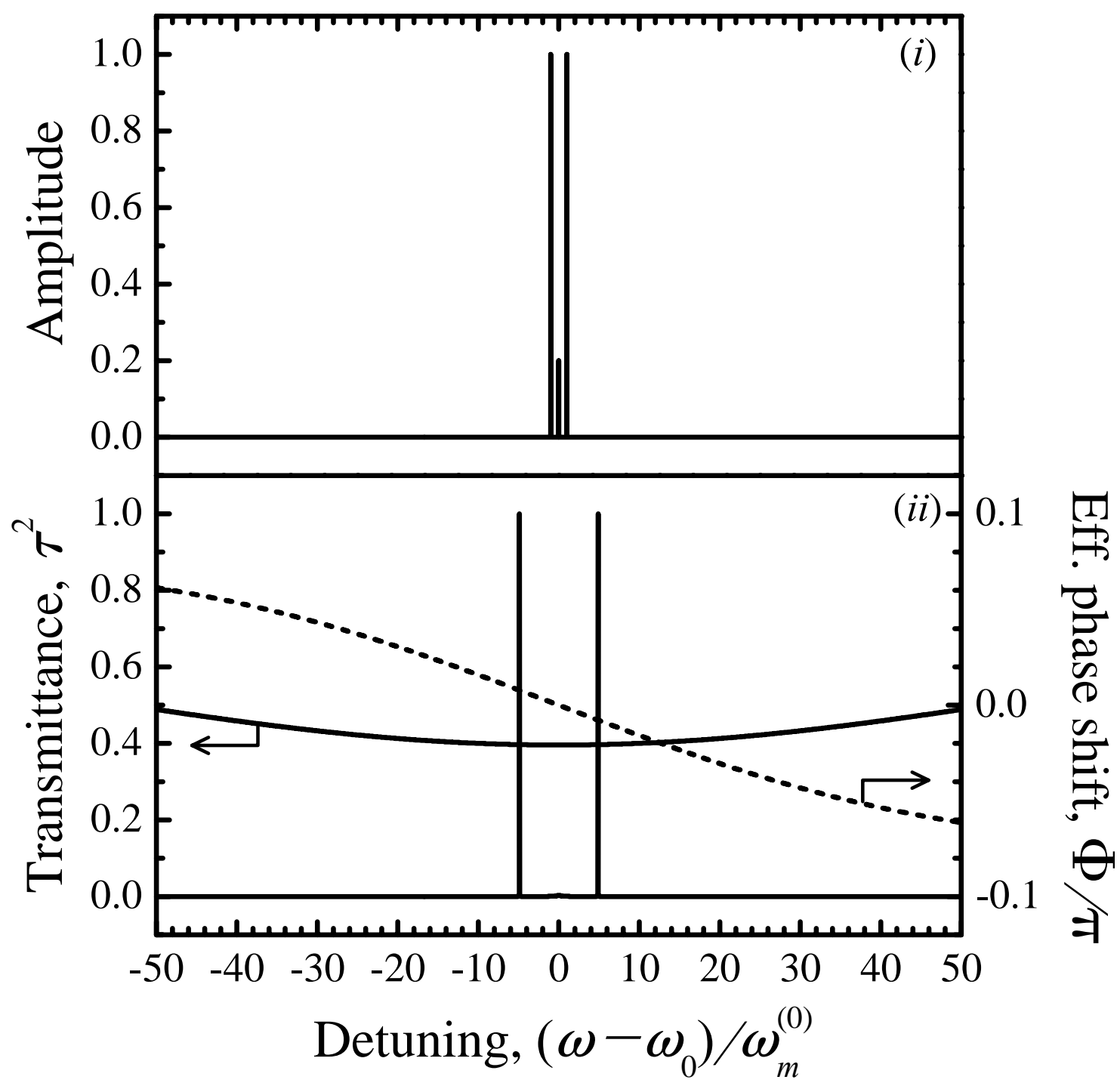

Figure 2a 
(b)

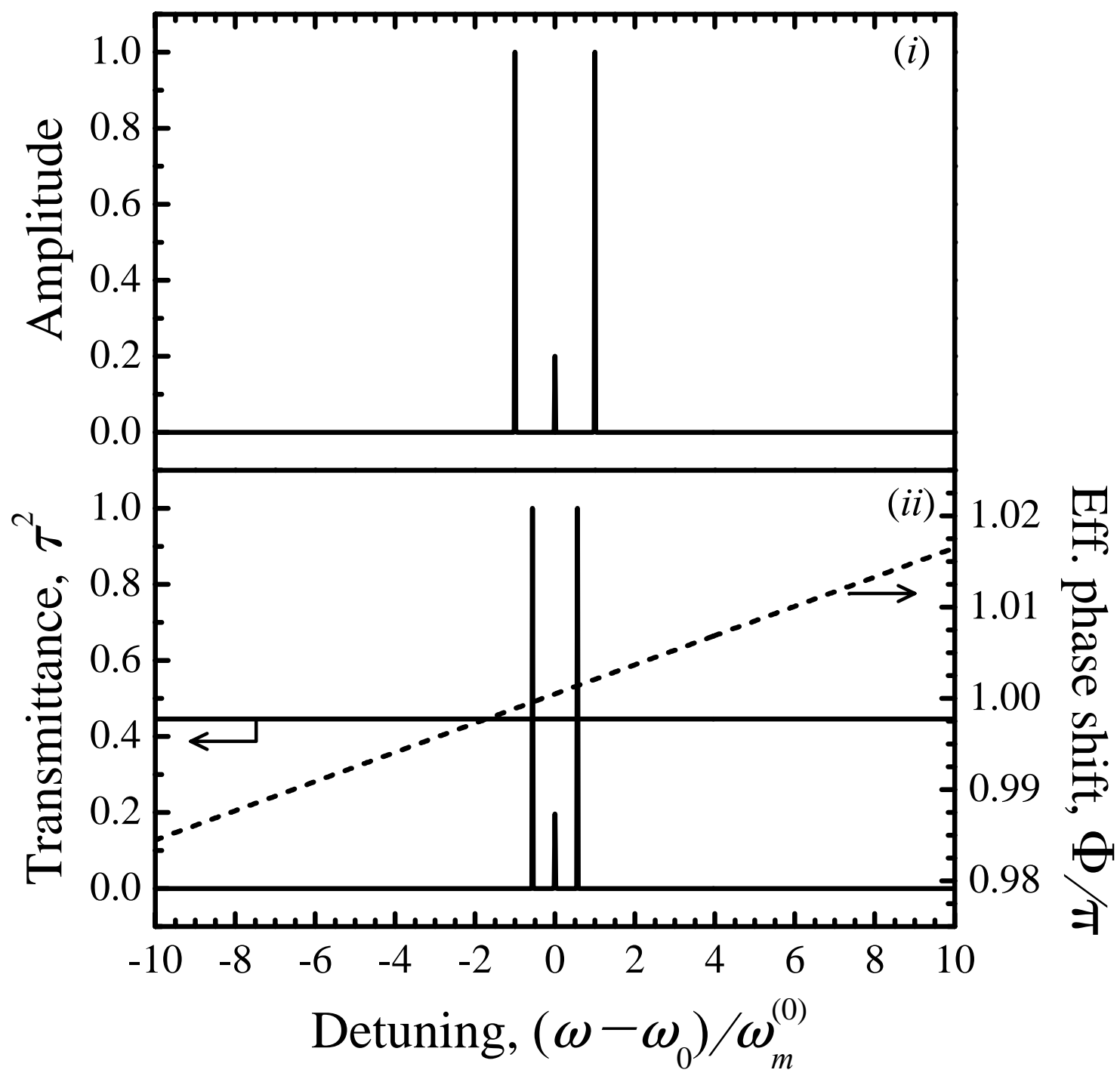

Figure 2b 


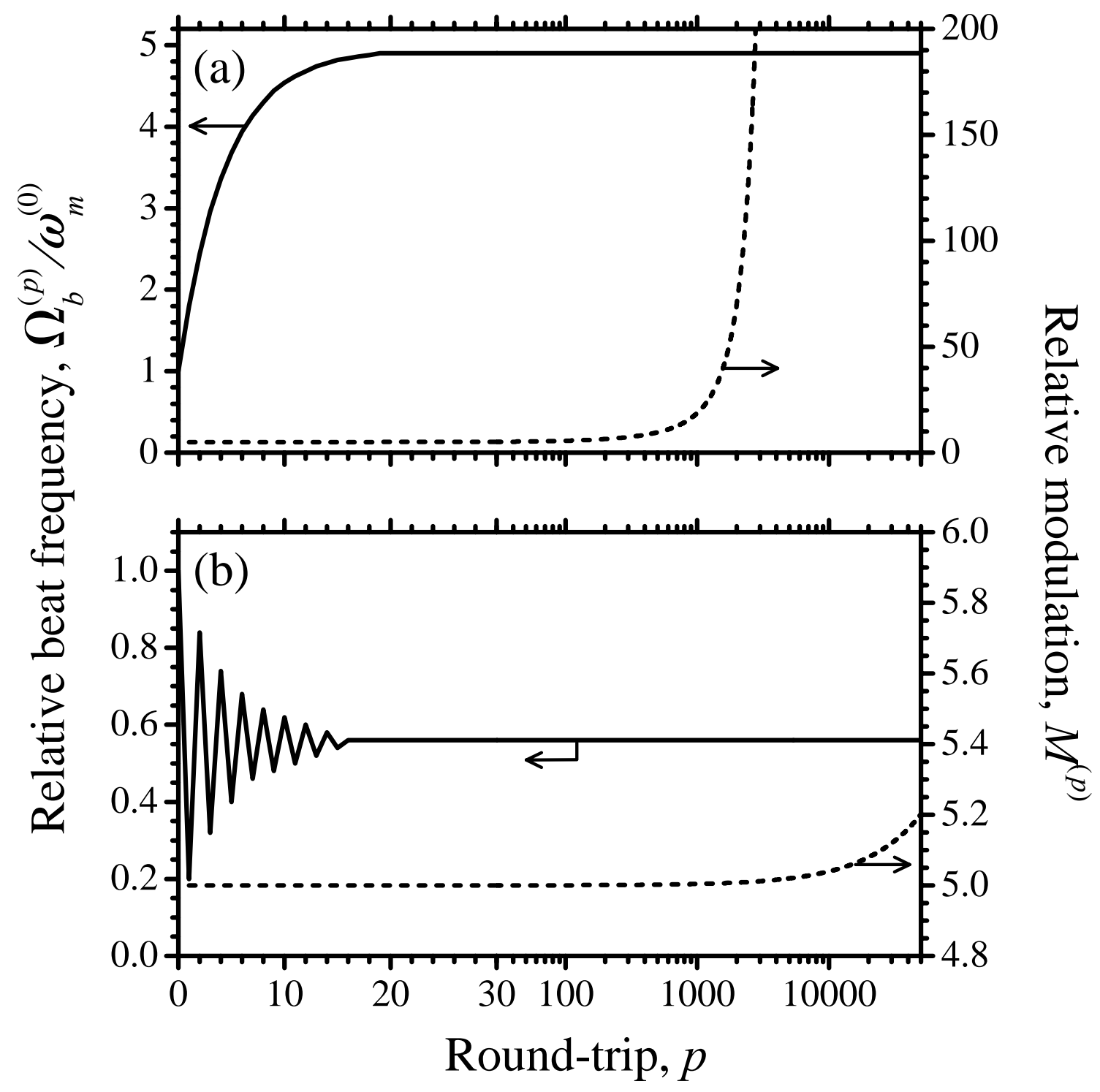

Figure 3 


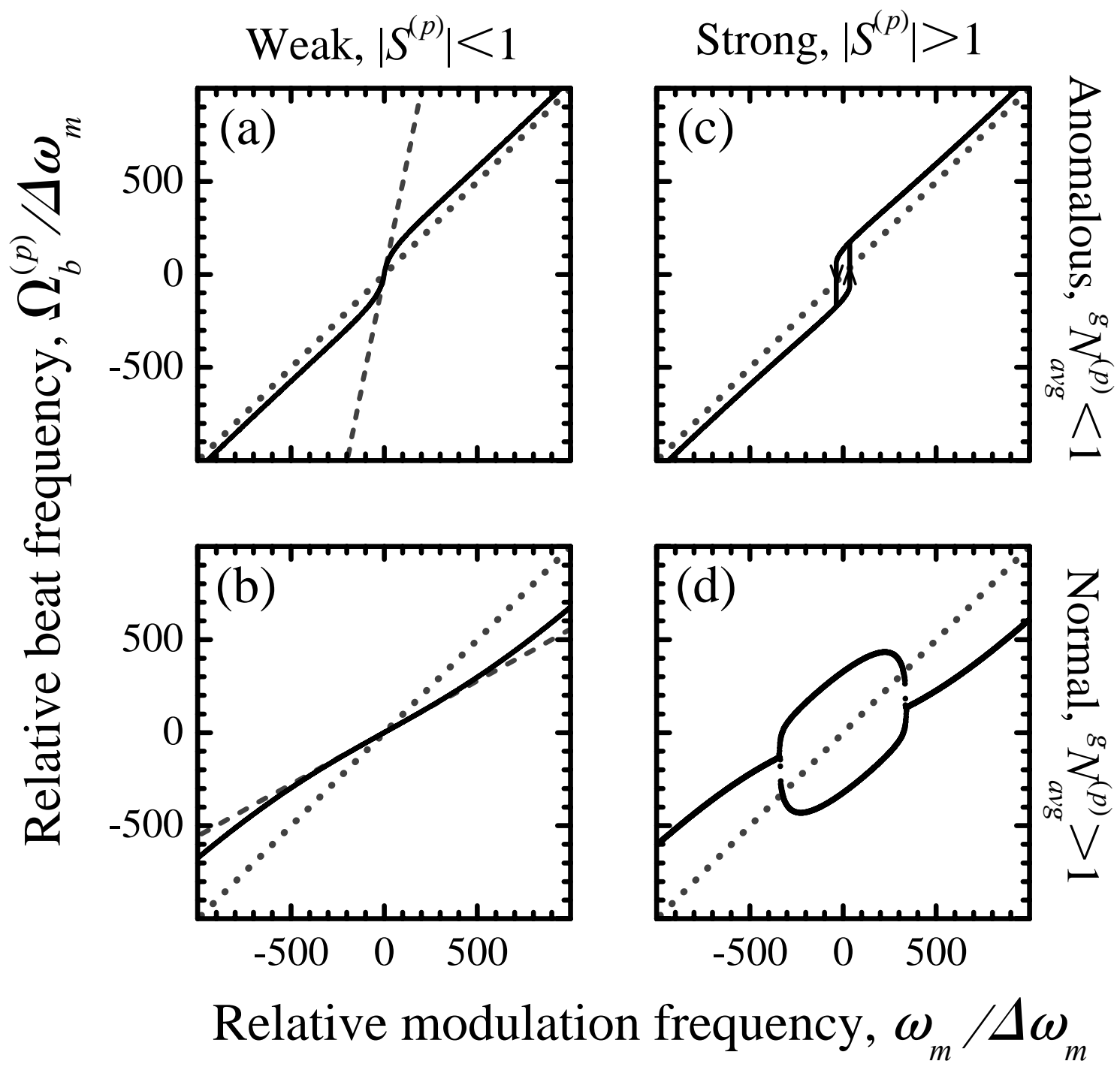

Figure 4 


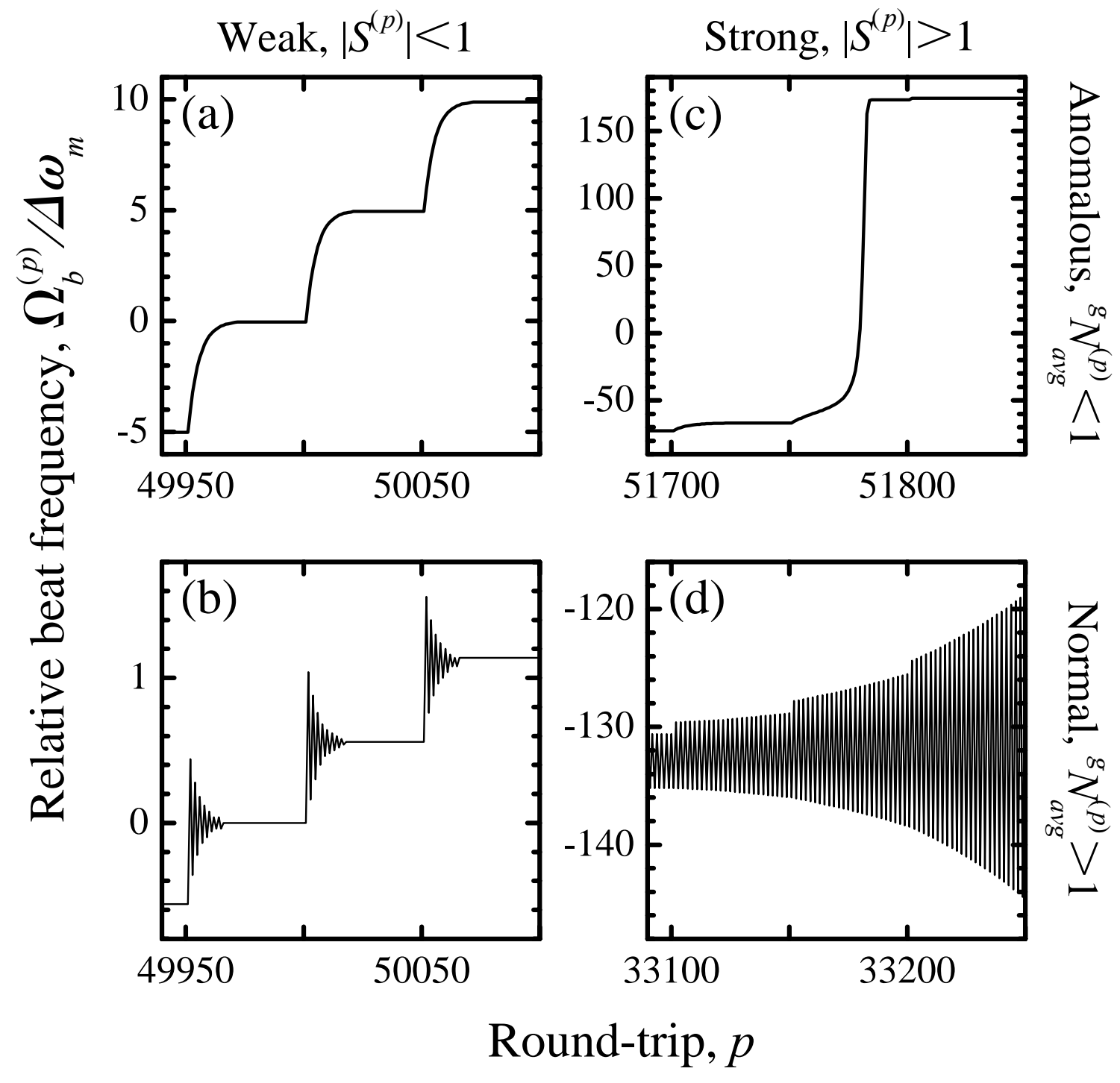

Figure 5 


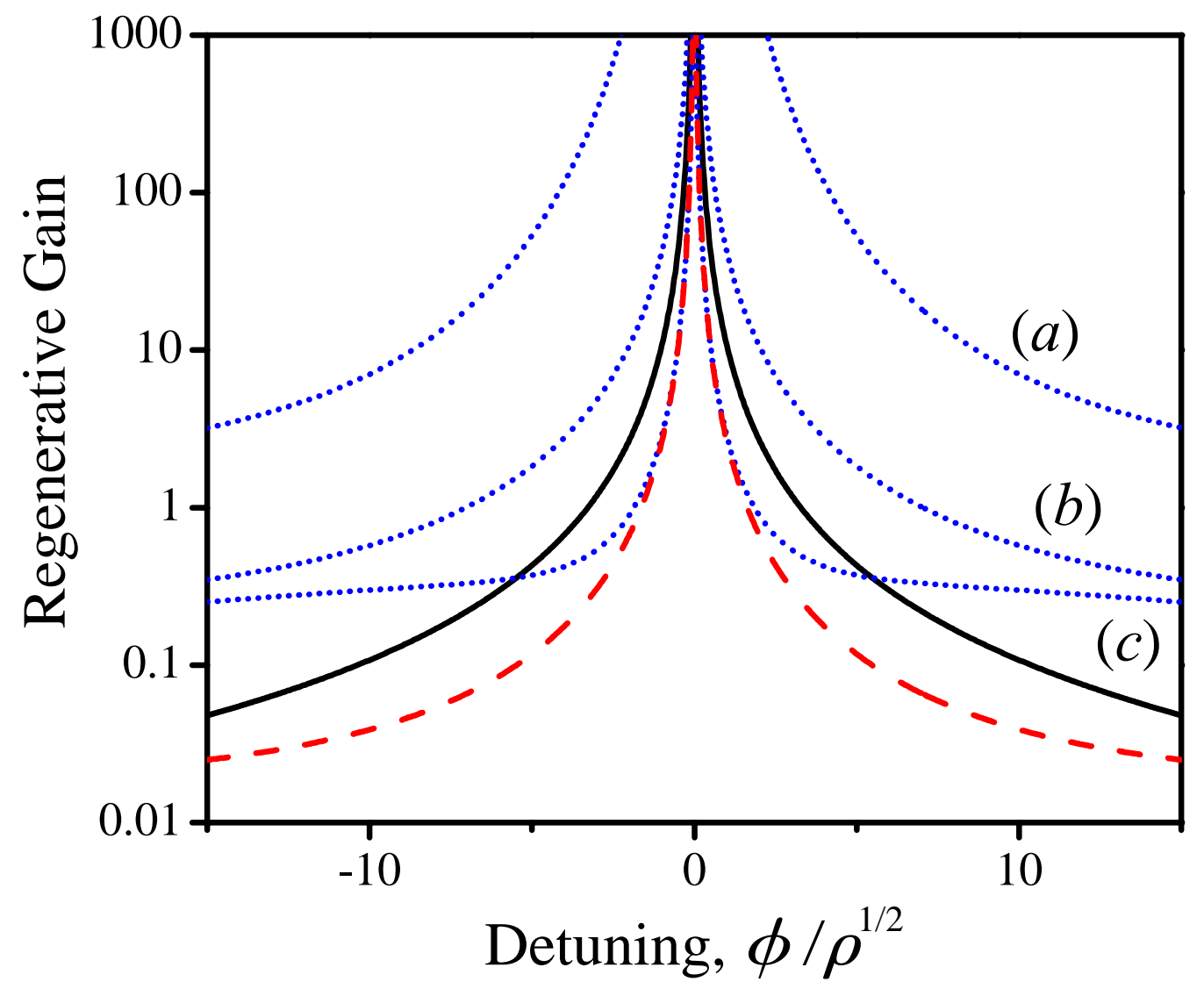

Figure 6 (color online) 


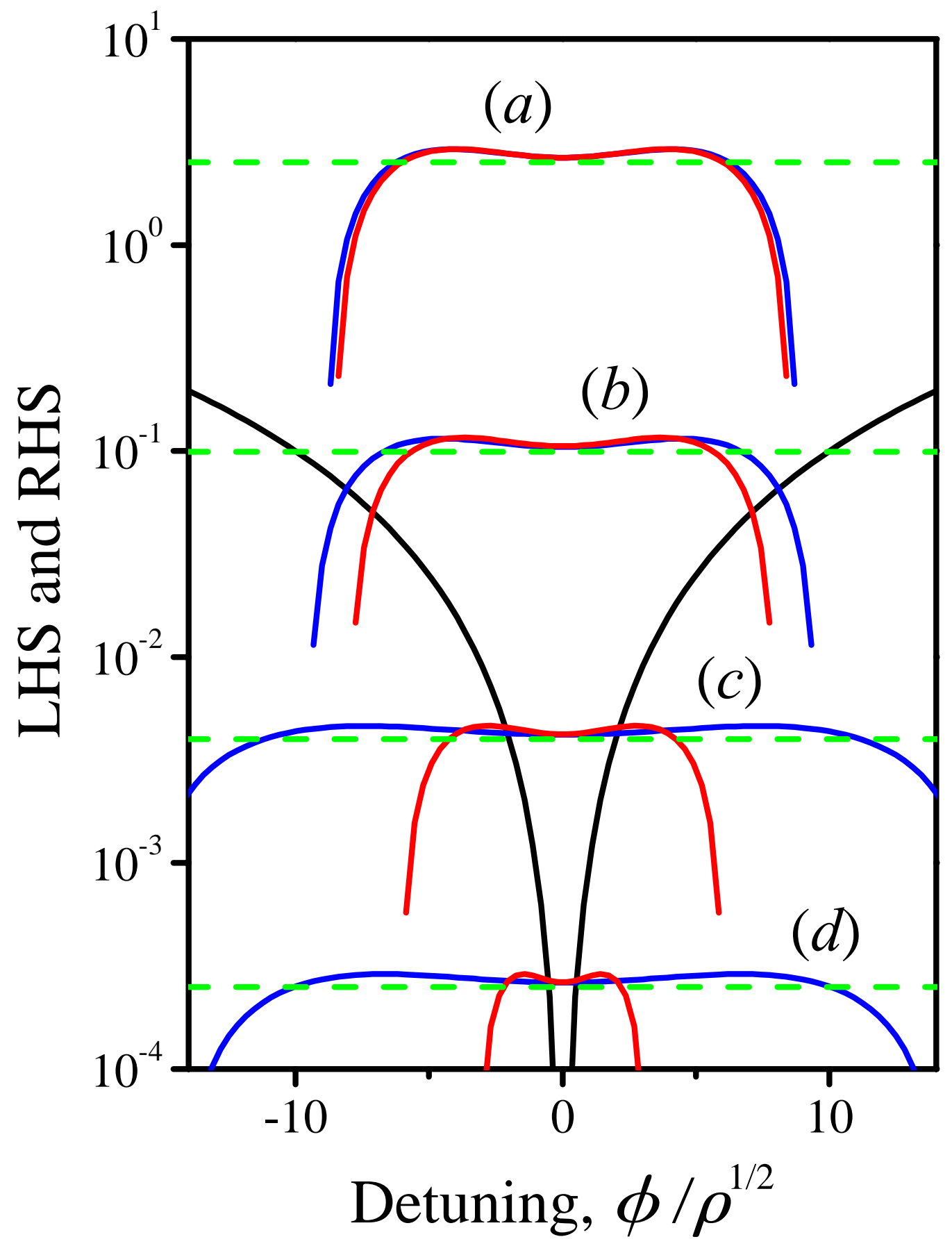

Figure 7 (color online) 


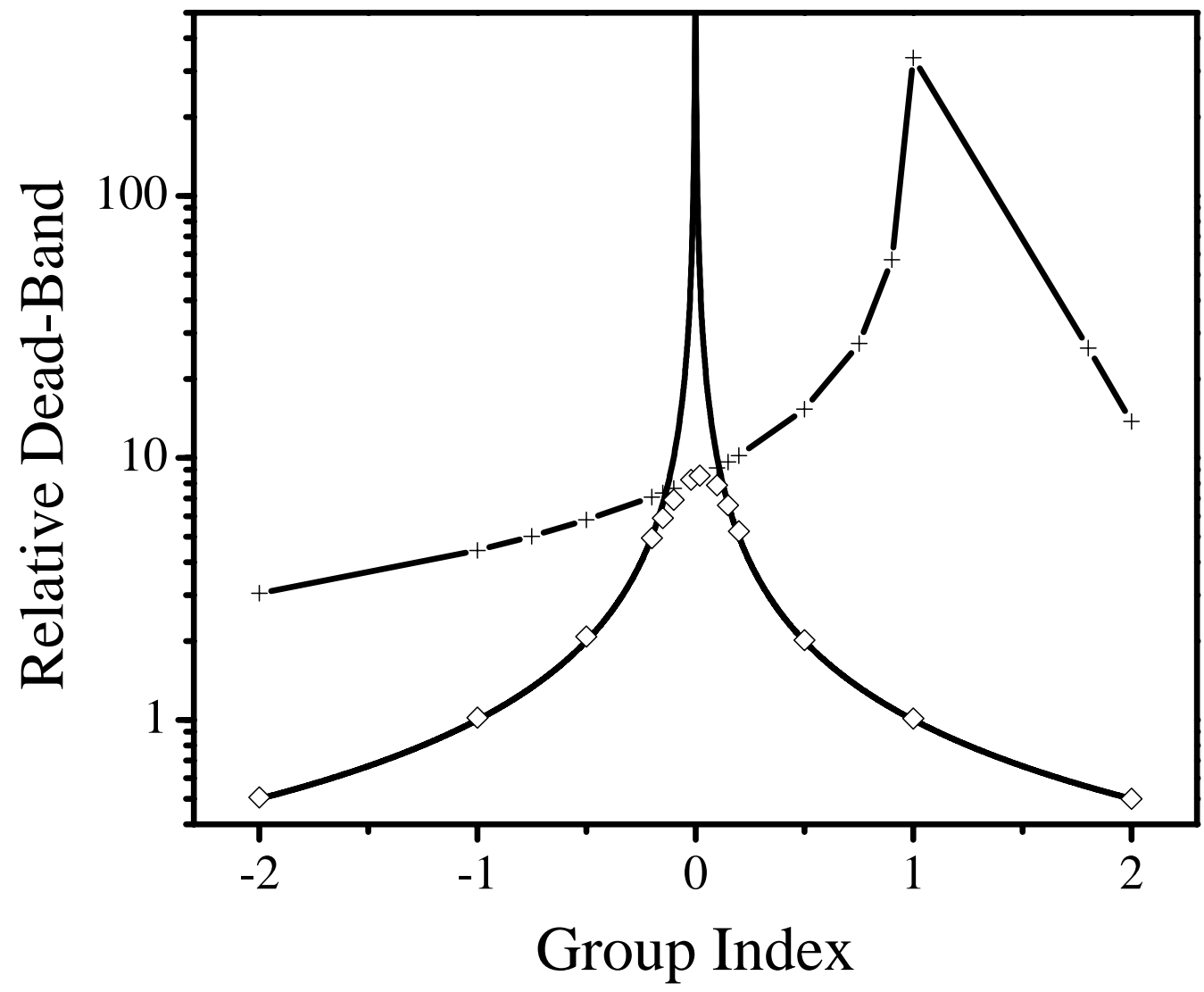

Figure 8 